\title{
Quantifying size-dependent interactions between fluorescently labeled polystyrene nanoparticles and mammalian cells
}

\author{
Juan A Varela ${ }^{1 *}$, Mariana G Bexiga ${ }^{1,2,3}$, Christoffer Åberg ${ }^{1}$, Jeremy C Simpson ${ }^{2}$ and Kenneth A Dawson ${ }^{1 *}$
}

\begin{abstract}
Background: Nanoparticles (NPs) are currently used in a wide variety of fields such as technology, medicine and industry. Due to the novelty of these applications and to ensure their success, a precise characterization of the interactions between NPs and cells is essential.

Findings: The current study explores the uptake of polystyrene NPs by 1321N1 human astrocytoma and A549 human lung carcinoma cell lines. In this work we show for the first time a comparison of the uptake rates of fluorescently labeled carboxylated polystyrene (PS) NPs of different sizes (20, 40 and $100 \mathrm{~nm}$ ) in two different cell types, keeping the number of NPs per unit volume constant for all sizes. We propose a reliable methodology to control the dose of fluorescently labeled NPs, by counting individual NPs using automated particle detection from 3D confocal microscopy images. The possibility of detecting individual NPs also allowed us to calculate the size of each nanoparticle and compare the fluorescence of single NPs across different sizes, thereby providing a robust platform for normalization of NP internalization experiments as measured by flow cytometry.
\end{abstract}

Conclusions: Our findings show that $40 \mathrm{~nm}$ NPs are internalized faster than $20 \mathrm{~nm}$ or $100 \mathrm{~nm}$ particles in both cell lines studied, suggesting that there is a privileged size gap in which the internalization of NPs is higher.

Keywords: Bio-nanotechnology, Cellular uptake, Nanoparticles, Single particle tracking

\section{Background}

Nanoparticles (NPs) can be defined as ultra fine particles with lengths between $1 \mathrm{~nm}$ to $100 \mathrm{~nm}$ in at least two of their dimensions. Currently nanomaterials are being used in a wide variety of applications such as engineering, food industry, cosmetics and medicine [1,2]. In medicine there are major expectations for the use of nanoparticles to facilitate targeted drug delivery [3-5]. Due to the novelty of these applications and to ensure their success, a precise characterization of the interactions between NPs and cells is essential.

Polystyrene (PS) NPs are widely used as a model to study interactions between NPs and cells due to various practical reasons including their commercial availability,

\footnotetext{
* Correspondence: juan.varela@cbni.ucd.ie; kenneth.a.dawson@cbni.ucd.ie ${ }^{1}$ Centre for BioNano Interactions, School of Chemistry and Chemical Biology, University College Dublin, Belfield, Dublin 4, Ireland

Full list of author information is available at the end of the article
}

high quality and wide variety of size and surface chemistry. These NPs have been reported to enter different cell types including hepatocytes [6], macrophages [7] and lung [8]. One general conclusion is that particles smaller than $100 \mathrm{~nm}$ are able to enter mammalian cells. The specific uptake pathways of these NPs, as well as the uptake rates, have been shown to be cell type- [9], NP size$[10,11]$ and shape-dependent [12] but are also related to the surface chemistry of the NP [13] and its hydrophobicity [14]. Although various studies indicate that the final localization of NPs is usually the lysosome $[7,15,16]$, the internalization mechanism is not fully understood [17]. Indeed, multiple mechanisms may intervene in parallel as we previously observed for both A549 and 1321N1 cells [18].

At present, most studies comparing the effects of the uptake of different sized NPs are based on exposure to 
the same concentration of particles measured in mass per unit volume. Using this approach, studies on the cellular uptake of different sized NPs $[19,20]$, as well as on their toxicity $[6,21]$, have shown size-dependent effects. Although this kind of particle exposure can provide useful information, it also presents clear drawbacks in the understanding of the interactions between cells and NPs, specifically in distinguishing whether the reported effects were due to the size or simply to a difference in NP number (e.g. there are two orders of magnitude between the actual number of $20 \mathrm{~nm}$ and $100 \mathrm{~nm}$ NPs when used at the same mass per unit volume concentration). These large differences in NP numbers may bias results when investigating toxicity, internalization, and intracellular traffic of different sized NPs, as these interactions are dose-dependent.

\section{Results and discussion}

In this study we used fluorescently labeled carboxylated $(-\mathrm{COOH})$ PS NPs (measuring 20, 40 and $100 \mathrm{~nm}$ in diameter) to understand the effects of size in their uptake by $1321 \mathrm{~N} 1$ and A549 cells. We began by carefully characterizing the NPs in phosphate buffered saline (PBS) at $\mathrm{pH} 7.0$ and $25^{\circ} \mathrm{C}$ as described in the Additional file 1: Experimental Section. The hydrodynamic size of the NPs in PBS was measured by dynamic light scattering (DLS), revealing that they were well dispersed in all cases (Table 1), as all measurements presented a low polydispersity index. The $\zeta$-potential of the NPs was also determined and as expected from the chemistry of the NPs, all displayed a negative $\zeta$-potential (Table 1), indicating that these NPs present a negative surface charge. NPs were also characterized in complete cell culture medium (Additional file 1: Table S1) revealing that they continued to be monodispersed, although the measured size was larger than that in PBS, most likely a consequence of the protein corona surrounding the NPs [22]. The $\zeta$-potential of the particles in cell culture medium was approximately zero, suggesting a screening effect of the NP surface charge due to the proteins present in the medium.

In order to expose cells to a fixed number of NPs we first estimated the number of NPs present in $1 \mathrm{~mL}$ of solution, at a certain concentration $C$ in $\mathrm{g} / \mathrm{mL}$. If the density of the particles is $\rho$ (in $\mathrm{g} / \mathrm{mL}$ ) and the diameter $d$ (in $\mu \mathrm{m}$ ), an estimation of the number of NPs/mL would be given by:

$$
\frac{\text { Number of particles }}{m L}=\frac{6 C \times 10^{12}}{\pi d^{3} \rho}
$$

However, the number of NPs/mL calculated in this way is only an approximation, as it relies on assumptions of both the stock concentration and the homogeneous particle size (see Additional file 1: Additional Information). In order to obtain a more precise measurement of the number of NPs present in solution we first established whether individual NPs could be identified from microscopy images. We therefore dispersed the NPs in $100 \%$ glycerol and imaged the NPs freely diffusing at $40^{\circ} \mathrm{C}$ in a spinning-disk confocal microscope. The high viscosity of glycerol (433 times more viscous than water at $40^{\circ} \mathrm{C}$ ) made NPs diffuse sufficiently slow such that it enabled reconstruction of reliable trajectories, even of the $20 \mathrm{~nm}$ NPs. Images were then processed with Imaris software by performing automated particle detection and subsequent tracking routines (Figure 1a). There are several other available software packages to track particles. We chose Imaris due to its good performance and three dimensional (3D) rendering capabilities, allowing easy visual inspection of the detection and tracking accuracy. Larger NPs $(100 \mathrm{~nm})$ could be tracked for a high number of frames without detectable bleaching (over 200 frames for $100 \mathrm{~nm}$ NPs), whereas $20 \mathrm{~nm}$ particles typically started to bleach after 50 to 100 frames. From the trajectories obtained, the mean square displacement (MSD) was calculated (see Additional file 1: Additional Information), and the diffusion coefficient derived from the plots of MSD versus time, as freely diffusing particles present a linear relation between the MSD and time. The Stokes-Einstein relation (Equation 2) applied to the diffusing particle was then used to obtain the radius of the particle $(a)$ :

$$
D=\frac{k_{B} T}{6 \pi \eta a}
$$

where $k_{\mathrm{B}}$ is Boltzmann's constant, $T$ is the temperature in Kelvin and $\eta$ the fluid (dynamic) viscosity. The MSD versus time plots for $20 \mathrm{~nm}, 40 \mathrm{~nm}$ and $100 \mathrm{~nm}$ PS-COOH NPs freely diffusing in glycerol (Figure 1b) allowed us to

\begin{tabular}{|c|c|c|c|}
\hline NP Denomination & Hydrodynamic Size [nm] & PDI [a] & $\zeta$-potential [mV] \\
\hline $20 \mathrm{~nm}$ & $33 \pm 1$ & 0.14 & $-27 \pm 3$ \\
\hline $40 \mathrm{~nm}$ & $44 \pm 1$ & 0.13 & $-24 \pm 3$ \\
\hline $100 \mathrm{~nm}$ & $114 \pm 4$ & 0.01 & $-34 \pm 2$ \\
\hline
\end{tabular}

Table 1 Dynamic light scattering characterization of PS NPs used

All particles were characterized in PBS at $\mathrm{pH} 7.0$ and $25^{\circ} \mathrm{C}$.

[a] Polydispersity Index. 


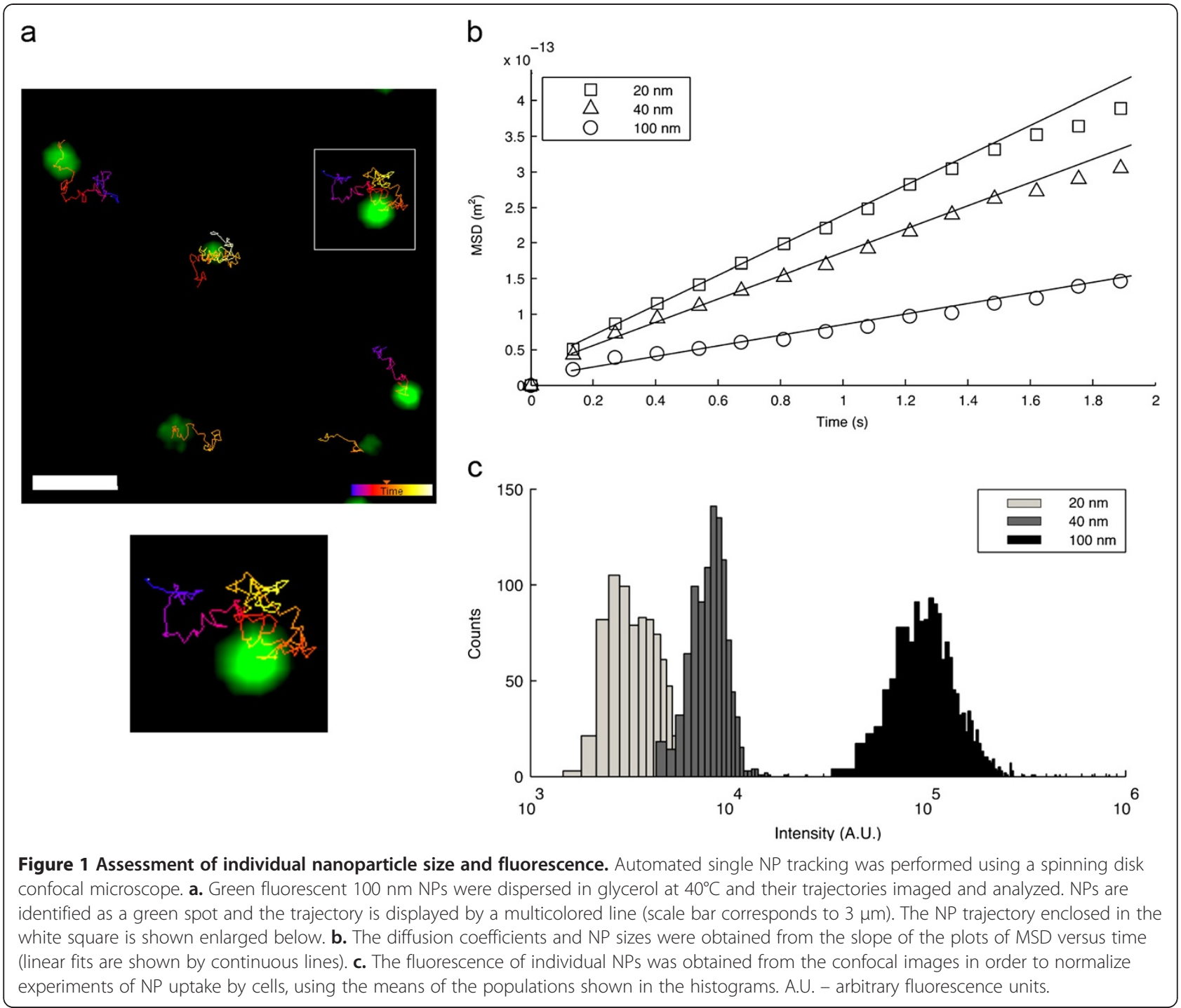

calculate the diffusion coefficients by fitting the data with first order polynomials. The particle size was then calculated using Equation 2. The sizes determined from the MSD analyses (Table 2) were very similar to those obtained by DLS (Table 1), thereby confirming that we were indeed observing individual NPs.

This allowed us to determine the absolute concentration of the NP stock in number of NPs per unit volume (Table 2). For this, a NP count was performed in glycerol from 3D images, each consisting of 50 confocal slices obtained with a spinning disk confocal microscope. After counting over 20,000 NPs for each case, and taking into account the volume of the z-stack obtained with the microscope, the number of NPs per unit volume in the dispersion, and therefore stock, was calculated (Table 2). The mean fluorescence of the detected NPs was also calculated (Figure 1c and Table 2) and this result was used to normalize subsequent experiments carried out

Table 2 Calculated numbers of PS NPs in stocks versus automatic count obtained from 3D confocal microscopy

\begin{tabular}{lcccr}
\hline NP Denomination & $\begin{array}{c}\text { SPT [a] calculated } \\
\text { size [nm] }\end{array}$ & $\begin{array}{c}\text { Estimated NP number } \\
{[\mathbf{N P} / \mathbf{m L}]}\end{array}$ & $\begin{array}{c}\text { Experimental NP count } \\
{[\mathbf{N P} / \mathbf{m L}]}\end{array}$ & $\begin{array}{c}\text { SPT Fluorescence Intensity } \\
\text { [A.U.] }\end{array}$ \\
\hline $20 \mathrm{~nm}$ & 32 & $4.5 \times 10^{15}$ & $2.2 \times 10^{15}$ & 1.0 \\
\hline $40 \mathrm{~nm}$ & 42 & $1.5 \times 10^{15}$ & $5.7 \times 10^{14}$ & 2.3 \\
\hline $100 \mathrm{~nm}$ & 92 & $3.6 \times 10^{13}$ & $3.7 \times 10^{13}$ & 31.0 \\
\hline
\end{tabular}

[a] Single particle tracking. 
in cells, by dividing flow cytometry fluorescence measurements by the mean fluorescence yield of each NP size. This normalization step takes into consideration that larger NPs are brighter than smaller ones. The number of detected NPs per unit volume was then compared with the estimated number of NPs per unit volume (using Equation 1) and we concluded that the values differed one fold for the 20 and $40 \mathrm{~nm} \mathrm{NPs}$, while for $100 \mathrm{~nm}$ NPs the experimental count coincided with the estimated number (Table 2). This is of extreme importance for biological experiments where it is necessary to use the same number of particles if accurate comparisons of phenotypic effects are to be made.

In order to quantify and study the kinetics of PS$\mathrm{COOH}$ NP uptake, cells were incubated with the different sized NPs for increasing lengths of time (1, 2, 3 and $4 \mathrm{~h}$ ) and the cell-associated fluorescence measured by flow cytometry. The arithmetic mean of the cell populations was compared across different samples, as the histograms of fluorescence intensity presented clear single peaks (Figure 2a, Additional file 1: Figure S1). Cells were exposed to $6 \times 10^{11} \mathrm{NPs} / \mathrm{mL}$ in complete cell culture medium for each NP type used. Analysis of the uptake kinetics for both cell lines showed that after a first transient non-linear regime (the control fluorescence corresponds to 0 value in the plots), the internalization of NPs was proportional to the incubation time of the experiment. To determine the rate of internalization of the NPs, we performed linear fits by least squares calculation for the interval between 1 and $4 \mathrm{~h}$ and determined the slope of the line, which corresponded to the rate of uptake. In order to compare across the different scenarios, all uptake rates were normalized to the uptake rate of the $20 \mathrm{~nm}$ nanoparticles in $1321 \mathrm{~N} 1$ cells. The uptake rate for $40 \mathrm{~nm}$ was 6.7 and for $100 \mathrm{~nm}$ NPs 3.8 (in arbitrary fluorescence units (A.U.) per hour, Figure 2b). For A549

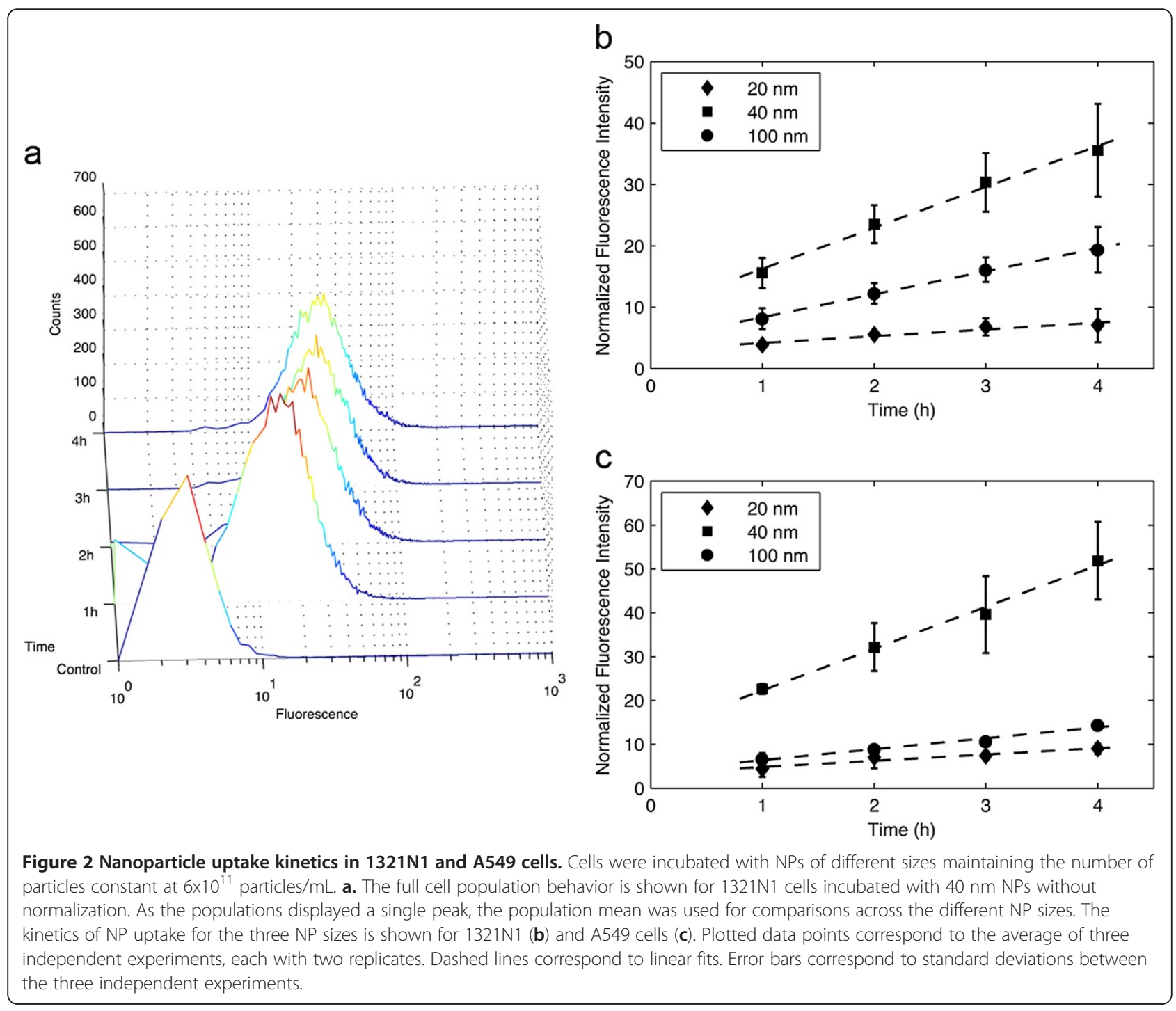


cells the rates of uptake of the NPs followed a similar trend, with the $20 \mathrm{~nm}$ NPs being those with the slowest uptake rate (1.4 A.U./h) and $40 \mathrm{~nm}$ NPs those that entered cells the most rapidly (9.5 A.U./h, Figure 2c). The uptake rate obtained for the $100 \mathrm{~nm}$ NPs $(2.5 \mathrm{~A} . \mathrm{U} / \mathrm{h})$ was slower than for $1321 \mathrm{~N} 1$ cells. As the cells were exposed to the same number of NPs, and the rates of uptake were different, these results may suggest that the mechanisms by which NPs of $40 \mathrm{~nm}$ or $100 \mathrm{~nm}$ are imported by the cell differ. It should be noted that the adhesion of larger NPs to the cell surface could be stronger than that of smaller NPs; e.g. the van der Waals force between a sphere and a surface is proportional to the diameter of the sphere [23]. Despite this, the uptake of $40 \mathrm{~nm}$ particles was faster than that of $100 \mathrm{~nm}$ particles, which might be explained by different kinetics of the endocytosis mechanisms utilized by the cells to internalize the NPs. In the case of the $20 \mathrm{~nm}$ NPs, van der Waals adhesion forces are smaller and the diffusion higher, which could contribute to the lower uptake rate when compared with the two larger NPs studied here.

In order to confirm that the PS-COOH NPs were being internalized by $1321 \mathrm{~N} 1$ and A549 cells and to investigate the intracellular trafficking of the NPs, we performed immunofluorescence for EEA1 and LAMP1, early endosomal and lysosomal markers, respectively, followed by confocal microscopy imaging. We studied the localization of the nanoparticles after $2 \mathrm{~h}$ of incubation with the same NP exposure conditions as for the flow cytometry experiments. These experiments revealed co-localization of NPs with both markers, suggesting that NPs follow the endosomal-lysosomal pathway inside cells (Additional file 1: Figures S2 and S3).

\section{Conclusions}

Our correlation between single particle tracking (SPT) analysis of NPs in solution and flow cytometry experiments measuring NP internalization proved to be a robust method to investigate bio-nano interactions. We observed that the number of NPs present in a particle stock may differ from the estimated numbers, and therefore careful characterization of the nanomaterials (including measuring the actual number of NPs) is important to quantitatively assess effects in biological environments. NP uptake rates were shown to differ between the two cell lines under study (Additional file 1: Figure S4) with the uptake rates being NP-size-dependent for each cell line. For both cell lines under study $40 \mathrm{~nm}$ NPs were internalized faster than $20 \mathrm{~nm}$ or $100 \mathrm{~nm}$ NPs, consistent with previous literature on gold nanoparticles [24]. This could suggest different internalization mechanisms for the different NP sizes, although this will need further clarification.

The ability to characterize NP dispersions at a single particle level provided us with the advantage of being able to obtain size-dependent NP uptake rates, that would have been meaningless if the cells had been exposed to different NP numbers. One clear strength of the presented method is that the NP dispersion is characterized in terms of size and fluorescence through SPT, yielding valuable information for the interpretation of results. The method is sufficiently simple that it can be routinely implemented prior to biological experiments investigating interactions between NPs and cells. One drawback, however, is that the fluorescently labeled NPs need to be sufficiently bright to be able to detect them individually. In our opinion, the standardization methodology that we describe here is appropriate for taking account of size effects of different NPs, and as such can be applied to a variety of experiments such as quantification of NP internalization, toxicity and trafficking. Furthermore, alternative experimental strategies such as keeping the total NP surface constant across different NP sizes could also be applied using this methodology. This work therefore provides a methodology that should be applicable to many future studies aiming to derive a greater and more quantitative understanding of the interactions between NPs and biological systems.

\section{Additional file}

\section{Additional file 1: Additional Information.}

\section{Abbreviations}

3D: 3 dimensional; A.U.: Arbitrary fluorescence units; DLS: Dynamic light scattering; MSD: Mean square displacement; NPs: Nanoparticles;

PBS: Phosphate buffered saline; PS: Polystyrene; SPT: Single particle tracking.

\section{Competing interests}

The authors declare that they have no competing interests.

\section{Authors' contributions}

JAV conceived, designed, performed and analyzed experiments and wrote the manuscript. MGB performed experiments and wrote the manuscript. C designed and analyzed experiments. JCS wrote the manuscript. KAD supervised and coordinated the project. All authors read and approved the manuscript.

\section{Acknowledgements}

We wish to thank Dr. Alfonso Blanco and the Flow Cytometry Core Facility from the UCD Conway Institute for all the assistance given. This work was supported by the EU FP6 project S.I.G.H.T (IST-2005-033700) and Science Foundation Ireland (SFI) RFP (09/RFP/MTR2425). The JCS lab is supported by a Principal Investigator award (09/IN.1/B2604) from SFI. MGB was supported by the Portuguese Fundação para a Ciência e Tecnologia

(SFRH/BD/15892/2005). CÂ was supported by Irish Research Council for Science, Engineering and Technology.

\section{Author details}

${ }^{1}$ Centre for BioNano Interactions, School of Chemistry and Chemical Biology, University College Dublin, Belfield, Dublin 4, Ireland. ${ }^{2}$ School of Biology and Environmental Science and Conway Institute of Biomolecular and Biomedical Research, University College Dublin, Belfield, Dublin 4, Ireland. ${ }^{3} \mathrm{PhD}$ Programme in Experimental Biology and Biomedicine, Centre for Neurosciences and Cell Biology, University of Coimbra, 3004-517 Coimbra, Portugal. 
Received: 12 April 2012 Accepted: 18 July 2012

Published: 24 September 2012

\section{References}

1. Dekkers S, Krystek P, Peters RJB, Lankveld DPK, Bokkers BGH, van Hoeven-Arentzen PH, Bouwmeester $\mathrm{H}$, Oomen AG: Presence and risks of nanosilica in food products. Nanotoxicology 2011, 5:393-405.

2. Osmond MJ, McCall MJ: Zinc oxide nanoparticles in modern sunscreens: an analysis of potential exposure and hazard. Nanotoxicology 2010, 4:15-41.

3. Pack DW, Hoffman AS, Pun S, Stayton PS: Design and development of polymers for gene delivery. Nat Rev Drug Discov 2005, 4:581-593.

4. Peer D, Karp JM, Hong S, Farokhzad OC, Margalit R, Langer R: Nanocarriers as an emerging platform for cancer therapy. Nat Nanotechnol 2007, 2:751-760.

5. Farokhzad OC, Langer R: Impact of nanotechnology on drug delivery. ACS Nano 2009, 3:16-20.

6. Johnston HJ, Semmler-Behnke M, Brown DM, Kreyling W, Tran L, Stone V: Evaluating the uptake and intracellular fate of polystyrene nanoparticles by primary and hepatocyte cell lines in vitro. Toxicol Appl Pharmacol 2010, 242:66-78.

7. Xia T, Kovochich M, Liong M, Zink Jl, Nel AE: Cationic polystyrene nanosphere toxicity depends on cell-specific endocytic and mitochondrial injury pathways. ACS Nano 2008, 2:85-96.

8. Geys J, Coenegrachts L, Vercammen J, Engelborghs Y, Nemmar A Nemery B, Hoet PHM: In vitro study of the pulmonary translocation of nanoparticles: a preliminary study. Toxicol Lett 2006, 160:218-226.

9. Foster KA, Yazdanian M, Audus KL: Microparticulate uptake mechanisms of in-vitro cell culture models of the respiratory epithelium. J Pharm Pharmacol 2001, 53:57-66.

10. Gaumet M, Gurny R, Delie F: Localization and quantification of biodegradable particles in an intestinal cell model: the influence of particle size. Eur J Pharm Sci 2009, 36:465-473.

11. Jiang W, Kim BYS, Rutka JT, Chan WCW: Nanoparticle-mediated cellular response is size-dependent. Nat Nanotechnol 2008, 3:145-150.

12. Gratton SEA, Ropp PA, Pohlhaus PD, Luft JC, Madden VJ, Napier ME, DeSimone JM: The effect of particle design on cellular internalization pathways. Proc Natl Acad Sci U S A 2008, 105:11613-11618.

13. Dausend J, Musyanovych A, Dass M, Walther $\mathrm{P}$, Schrezenmeier $\mathrm{H}$, Landfester K, Mailänder V: Uptake mechanism of oppositely charged fluorescent nanoparticles in HeLa cells. Macromol Biosci 2008, 8:1135-1143.

14. Lorenz S, Hauser CP, Autenrieth B, Weiss CK, Landfester K, Mailänder V: The softer and more hydrophobic the better: influence of the side chain of polymethacrylate nanoparticles for cellular uptake. Macromol Biosci 2010, 10:1034-1042.

15. Xiao Y, Forry SP, Gao X, Holbrook RD, Telford WG, Tona A: Dynamics and mechanisms of quantum dot nanoparticle cellular uptake. J Nanobiotechnol 2010, 8:13.

16. Clift MJD, Rothen-Rutishauser B, Brown DM, Duffin R, Donaldson K, Proudfoot L, Guy K, Stone V: The impact of different nanoparticle surface chemistry and size on uptake and toxicity in a murine macrophage cell line. Toxicol Appl Pharmacol 2008, 232:418-427.

17. Iversen T-G, Skotland T, Sandvig K: Endocytosis and intracellular transport of nanoparticles: Present knowledge and need for future studies. Nano Today 2011, 6:176-185.

18. Dos Santos T, Varela J, Lynch I, Salvati A, Dawson K: Effects of transport inhibitors on the cellular uptake of carboxylated polystyrene nanoparticles in different cell lines. PLOS ONE 2011, 6:e24438.

19. Zauner W, Farrow NA, Haines AM: In vitro uptake of polystyrene microspheres: effect of particle size, cell line and cell density. J Control Release 2001, 71:39-51.

20. Shapero K, Fenaroli F, Lynch I, Cottell DC, Salvati A, Dawson KA: Time and space resolved uptake study of silica nanoparticles by human cells. $\mathrm{Mol}$ Biosyst 2011, 7:371-378.

21. Oh W-K, Kim S, Choi M, Kim C, Jeong YS, Cho B-R, Hahn J-S, Jang J: Cellular uptake, cytotoxicity, and innate immune response of silica-titania hollow nanoparticles based on size and surface functionality. ACS Nano 2010, 4:5301-5313.

22. Cedervall T, Lynch I, Lindman S, Berggård T, Thulin E, Nilsson H, Dawson KA, Linse S: Understanding the nanoparticle-protein corona using methods to quantify exchange rates and affinities of proteins for nanoparticles Proc Natl Acad Sci U S A 2007, 104:2050-2055.

23. Israelachvili J: Intermolecular and Surface Forces. 2nd edition. London: Academic Press; 1992

24. Chithrani BD, Ghazani A, Chan WCW: Determining the size and shape dependence of gold nanoparticle uptake into mammalian cells. Nano Lett 2006, 6:662-668.

doi:10.1186/1477-3155-10-39

Cite this article as: Varela et al:: Quantifying size-dependent interactions between fluorescently labeled polystyrene nanoparticles and mammalian cells. Journal of Nanobiotechnology 2012 10:39.

\section{Submit your next manuscript to BioMed Central and take full advantage of:}

- Convenient online submission

- Thorough peer review

- No space constraints or color figure charges

- Immediate publication on acceptance

- Inclusion in PubMed, CAS, Scopus and Google Scholar

- Research which is freely available for redistribution 